\title{
A "HOLTER" FOR PARKINSON'S DISEASE: VALIDATION OF THE ABILITY TO DETECT ON-OFF STATES USING THE REMPARK SYSTEM
}

\section{Authors}

Àngels Bayés $\mathrm{MD}, \mathrm{PhD}^{1}$, Albert Samá $\mathrm{PhD}^{2}$, Anna Prats $\mathrm{MSc}^{11}$, Carlos Pérez-López $\mathrm{PhD}{ }^{3}$, Maricruz Crespo-Maraver $\mathrm{PhD}^{1,4}$, Juan Manuel Moreno $\mathrm{PhD}^{3}$, Sheila Alcaine ${ }^{1}$, Alejandro Rodriguez-Molinero MD, $\mathrm{PhD}^{5,6}$, Berta Mestre ${ }^{1}$, Paola Quispe ${ }^{1}$, Ana Correia de Barros PhD ${ }^{7}$, Rui Castro PhD ${ }^{7}$, Alberto Costa ${ }^{8}$, Roberta Annicchiarico MD, PhD ${ }^{9}$, Patrick Browne ${ }^{10}$, Tim Counihan $M^{11}$, Hadas Lewy ${ }^{12}$, Leo R Quinlan ${ }^{13}$, Dean Sweeney ${ }^{14}$, Gearóid ÓLaighin ${ }^{13}$, Jordi Rovira ${ }^{15}$, Daniel Rodriguez-Martin ${ }^{3}$, and Joan Cabestany PhD $^{3}$

${ }^{1}$ Centro Médico Teknon - Grupo QuironSalud, Parkinson Unit. Barcelona, Catalunya, ES ${ }^{2}$ Universitat Politècnica de Catalunya, Automatic Control Department. Vilanova I la Geltrú, Catalunya, ES

${ }^{3}$ Universitat Politècnica de Catalunya, CETpd. Vilanova I la Geltrú, Catalunya, ES ${ }^{4}$ Fundació Althaia, Divisió de Salud Mental. Manresa, Catalunya, ES ${ }^{5}$ Consorci Sanitari del Garraf, Clinical Research Unit. Vilanova I la Geltrú, Catalunya, ES ${ }^{6}$ National University of Ireland, Galway, School of Engineering and Informatics. Galway, IE. ${ }^{7}$ Associaçao Fraunhofer Portugal Research, Fraunhofer Portugal AICOS (FhP-AICOS). Porto, PT ${ }^{8}$ Niccolò Cusano University, Psychology. Rome, IT ${ }^{9}$ Foundazione Santa Lucia, Technology-assisted neuro-rehabilitation laboratory. Rome, IT ${ }^{10}$ University Hospital Galway, Neurology Department. Galway, IE.

${ }^{11}$ National University of Ireland, Galway. Faculty of Medicine, Neurology. Galway, IE.

${ }^{12}$ Maccabi Heathcare Services. International center for R\&D. Tel-Aviv. IL.

${ }^{13}$ National University of Ireland, Galway. Electrical \& Electronic Engineering. Galway, IE.

${ }^{14}$ National University of Ireland, Galway. Physiology, School of Medicine. Galway, IE.

${ }^{15}$ Telefonica, R\&D. Barcelona, Catalunya, ES

Correspondence to:

Àngels Bayés

Unidad de Parkinson y Trastornos del Movimiento

Centro Médico Teknon.

Pso. Bonanova 26, Barcelona, Spain. 08022.

Email: 11741abr@comb.cat

Tel: +34 9341755 86/ Fax: +34 934175566

Full Length Article Word Count: 3475 words

Abstract Word Count: 194 words

Running Title: A "HOLTER" TO DETECT ON-OFF STATES

Key words: Parkinson's disease, on-off fluctuations, motor complications, REMPARK system, wearable sensor, and automatic assessment. 
Abstract: The treatment of Parkinson's disease (PD) with levodopa is very effective. However, over time, motor complications (MCs) such as ON-OFF fluctuations appear, restricting the patient from leading a normal life. Gathering accurate information about the clinical status of the patient is essential for planning treatment and assessing its effect. Systems such as the REMPARK system, capable of accurately and reliably monitoring ON-OFF fluctuations, are of great interest. Objective: To validate the ON-OFF detection function through the REMPARK System. Methods: Forty-one patients with moderate to severe idiopathic PD were recruited. Patients with motor fluctuations, freezing of gait and/or dyskinesia and who were able to walk unassisted in the OFF phase, were included in the study. Patients wore the REMPARK System for 3 days and completed a diary of their motor state once every hour. Results: The record obtained by the REMPARK System, compared with patient-completed diaries, demonstrated 97\% sensitivity in detecting OFF states and $88 \%$ specificity. Conclusion: The REMPARK System detects an accurate evaluation of ON-OFF fluctuations in PD; this technology paves the way for an optimisation of the symptomatic control of PD motor symptoms as well as an accurate assessment of medication efficacy. 


\section{INTRODUCTION}

Parkinson's disease (PD) is complicated over time by both motor complications (MCs)[1] and non-motor complications (NMCs)[2]. Symptomatic control of MCs can be challenging and requires frequent adjustment of therapy on account of unpredictable therapeutic responses. Gathering accurate information about a patient's condition throughout the day is essential to plot the optimal treatment plan. In clinical practice, the only method currently available is based on diaries filled in by patients and their caregivers, recording hours of ON-OFF and the presence of dyskinesias[3]. However, this method has limitations that make it unreliable, such as motor and cognitive difficulties impeding the ability to complete the diary, and the subjective nature of the self-evaluation[4]. Hence, there is considerable research interest in potential solutions that can improve disease management[5-8]. Wearable inertial sensors, have been widely used to analyse symptoms of PD such as tremor, bradykinesia, or dyskinesia. Some studies have examined the monitoring of motor states, but the results demonstrated only modest sensitivity and specificity[9-11]. Other studies used more than one sensor[12-16], resulting in less comfort for the patient. Moreover, monitoring was performed in controlled clinical environments, where conditions are different to patients' daily life[17]. The REMPARK system (REMPARK Personal health device for the remote and autonomous management of Parkinson's disease, FP7 project REMPARK ICT-287677) is a wearable system, developed between 2011-2015, to monitor motor states through a system requiring a single sensor. Analogous to the "Holter" for the monitoring of cardiac parameters, the REMPARK System is designed to record the patient's condition during daylight hours and to aid in managing the disease via the smartphone. The object of this study is to validate the REMPARK System as a system for automatic registration of ON-OFF fluctuations associated with PD treatment.

\section{METHODS}

\section{$\underline{\text { Subjects }}$}

A prospective pilot study was conducted, in which the recruitment was carried out following a convenience sampling among patients from the different centres participating in the REMPARK project: UParkinson-Teknon, (Barcelona), Fondazione Santa Lucia (Rome), Maccabi HealthcareServices (Tel Aviv) and the National University of Ireland Galway (Galway). The investigation was conducted in accordance with the Helsinki declaration 1964 (2008 Revision) and Good Clinical Practice guidelines. Institutional Review Board (IRB) approval was obtained from each clinical centre. All participants gave written informed consent and the following inclusion criteria were applied: a clinical diagnosis of idiopathic PD[18]; motor fluctuations, freezing of 
gait and/or dyskinesia; able to walk unassisted in the OFF phase; between 50 and 80 years of age and the ability to understand the potential risks and benefits of the study.

Subjects were excluded if they had other health problems that hampered physical activity and gait, were unable to recognise the ON-OFF fluctuations after proper training, met the criteria for dementia, according to DSM-IV-TR[19], were unwilling to cooperate, or were participating in other clinical trials.

\section{Equipment, variables and instruments}

\section{Assessment Instruments}

Patients' full clinical histories were collected. Standardised neurological assessment included the Unified Parkinson's Disease Rating Scale (UPDRS)[20] and Hoehn \& Yahr (H\&Y) staging[21]. Patients also completed a questionnaire to detect motor fluctuations and dyskinesia, and the Mini-Mental State (MMSE)[22] to evaluate cognitive function. Usability was assessed by using the Usability Scale (SUS)[23], and the QUEST-questionnaire[24] to evaluate satisfaction.

\section{Equipment}

The equipment used in the study consisted of the wearable elements of the REMPARK is described in a previous article [25].

\section{Design and procedures}

This is a prospective transversal pilot study, in which each participant wears the REMPARK System under real conditions during 3 consecutive days. The study took 2 extra days to make individual adjustments and adaptations (figure 1).

The first stage (day 0) focused on the recruitment of the subjects. Socio-demographic data, neurological data and written informed consent, were collected from all subjects enrolled in the study. The second phase (day 1) consisted in training patients and caregivers on how to operate the REMPARK System and the method for recording motor status in the diary. In the third phase (days 2, 3 and 4), patients used the system in their daily life for a minimum of 8 hours a day. In the diary, they recorded their motor status once every hour, timing of medication doses and their sleeping hours. A researcher went to the patient's home once a day and reviewed the proper functioning of the system. They administered the UPDRS part III to analyse the correlation between patient diary annotations and the actual motor status. In addition, approximately every two hours, the researchers called patients to verify that the system was working and asked about their motor state at that time. The motor state recorded by researchers in these follow-up calls was compared with that scored by patients in the diary. 
In case of discrepancy, the corresponding period was excluded from the analysis. At the end of the $4^{\text {th }}$ day, patients answered the usability and satisfaction questionnaires $[23,24]$ and reported on additional technical incidences.

\section{REMPARK System-On-Off Detection Algorithms}

The estimation of the motor state is based on a set of real-time algorithms applied to the sensor. A first algorithm analyses gait[26] and a second one analyses dyskinesia[27]. A third algorithm merges the information of the first two, providing the motor state estimation (see Appendix)

\section{Validation against ON-OFF diaries}

The ON-OFF diaries completed by the patients were used as the gold standard. Given that diaries are known to lack reliability[4], we developed a number of strategies that would improve their validity:

Firstly, the validity of diaries was evaluated based on clinicians' expertise through variations in the UPDRS motor score. The validity of the gold standard has been evaluated based on an objective measure consisting in the correlation between UPDRS scores and motor states provided by patients at the time of diary recording. Motor state is represented with $2=0 F F$, $1=$ Intermediate and $0=\mathrm{ON}$, so positive correlations are expected. Patients whose Pearson correlation coefficient was lower than 0.2 were not included in the study, since their ON-OFF diaries were considered not reliable to be used as gold standard.

Secondly, motor states recorded by the researchers during calls were compared with the notes made by patients in their diaries. In case of discrepancy, the corresponding periods were excluded from the analysis.

Thirdly, in order to consider a diary motor state record valid, it must appear two consecutive times in the diary, so that the patient demonstrates some consistency over a two-hour epoch. In the event of a motor state changing between two consecutive records, both records were excluded from the analysis, since the time in which the motor state changed is considered nonreliable.

\section{$\underline{\text { Data Analysis }}$}

Motor state estimations of the REMPARK System were compared with the annotations made by the patients. The comparison is done in terms of specificity and sensitivity for both ON and OFF motor states. Intermediate states have not been evaluated to ensure the validity of the reported motor state and because many patients only reported ON and OFF states. 
Consequently, REMPARK System validation is done as a binary diagnostic test in which positive cases correspond to reported OFF annotations and negative cases to the ON ones. This way, the accuracy in detecting OFF states is reported as sensitivity and the accuracy in ON states is given as specificity.

\section{RESULTS}

Fifty-four patients were initially contacted, 44 of whom met inclusion criteria and agreed to participate. Three of these patients did not complete the study procedure. The first due to lack of adherence to the study protocol and the second due to a health condition which required hospitalisation, not related to study devices or procedures. Forty-one patients completed the study days and evaluations (Figure 2). Table 1 shows detailed data of the characteristics of the participants. Of the 41 patients who completed the study, the data for 5 of them could not be analysed because the diary data was incomplete or unavailable. In addition, data from 3 patients was removed due to a low Pearson correlation coefficient between UPDRS scores and the motor state reported by the patient (correlations: $-0.62,0.06$ and 0.2 , respectively). Data from 33 patients were analysed.

The average specificity and sensitivity achieved by the system in recognising ON-OFF motor states is $88 \%$ and $97 \%$ respectively. The system generated an average of 26.3 hourly-based motor state detections per patient over the 3 days (9.6 ON, 3.5 OFF and 13.2 in intermediate state). Thus, on average, for each of the three days, almost 9 hours of monitoring were provided by the system per day. However, only those hours in which the patient reporting an ON or an OFF motor state coincided with the REMPARK system estimation were used. This resulted in validating 5 hours per patient (Table 2).

Health-safety of the REMPARK System: During the experimental period, 7 participants presented "adverse events" on health. One of them had a "serious adverse event" that required hospitalisation. The most frequent adverse events were limb pain $(n=3)$ and depressive symptoms $(n=3)$. Neck pain was also reported $(n=1)$. All adverse events, were considered as unrelated to the investigational device.

Usability and user satisfaction: The median score of SUS in this study was 70 (IQR 25). A SUS score of more than 50 is considered acceptable, and above 68-70 good [23].In the QUESTquestionnaire[24], no patient was "not satisfied at all" with the system. Five per cent were "not very satisfied", $20 \%$ "more or less satisfied" and $76 \%$ "satisfied" or "very satisfied". Comfort is the element with the lowest score and security with the highest. 


\section{DISCUSSION}

Systems that monitor PD symptoms reliably and accurately can be very useful in optimising treatment in patients. Our study evaluated the accuracy of the REMPARK system for monitoring ON-OFF states in Parkinson's patients in real conditions.

Despite the widespread use of patient diaries both in clinical practice and research settings for monitoring motor function, their limitations and subjectivity are well documented[28]. In our study we attempted to improve the reliability of the data obtained from the diaries (gold standard). Although this strategy reduces the number of patients' records used, it ensures that the records employed to validate the system are reliable. When applying this method, the accuracy in detecting the ON state (specificity), achieves $88 \%$ and in detecting OFF states $97 \%$ (sensitivity). However, our findings need to be replicated in other cases, given the great clinical variation of patients affected by PD. An important aspect of the study is that the results were obtained under real life conditions, albeit those studies are very challenging, and may entail false positive or negative readings.

One of the limitations of the system is that in order to assess the motor state, the patient must be walking or exhibit dyskinesias. Another limitation is the restricted sample size. However, because the average number of monitored hours is approximately 9 , we consider that the number of detections made is sufficient to register the motor state.

One of the main advantages in the detection of the motor state by the REMPARK system is that patients need to wear only a single sensor, making it compatible with an active life. In accordance with the results obtained in other studies[29], participants in our study also considered it to be user-friendly and were satisfied with the system. Although this is a very encouraging result, again it must be considered with some caution since the questionnaires were not anonymous.

The data obtained in this study show that the REMPARK system is able to detect fluctuations in patients' ON-OFF state. Given the increasing interest in this research topic, if the data is confirmed by further studies, it could prove to be an alternative tool to patients' diaries, it being more reliable as it is not subject to possible bias and could also empower patients. Furthermore, the system paves the way for its clinical use in the future in related areas: the automatic control of continuous infusion pumps or to Deep Brain Stimulation management, the automatic application of external cues to guide the gait or for comprehensive and reliable analysis of changes in motor states. Additionally, it could contribute positively to the quality of 
life of PD patients[30]. In this way, further studies on a larger sample would be of interest to show improvement in quality of life after treatment optimisation by the REMPARK System.

\section{CONCLUSIONS}

There is a need for a reliable tool to detect a patient's motor-state, in order to adjust therapy. This paper has presented the validation of the REMPARK system for detecting the ON-OFF states with a high degree of accuracy. The reliable detection of the motor state of PD patients throughout the day can dramatically change the value of clinical drug trials. Furthermore, it allows for individual adjustment of symptomatic treatment with a high degree of accuracy. 


\section{AUTHOR ROLES}

1) Research project: A. Conception, B. Organization, C. Execution; 2) Statistical Analysis: A. Design, B. Execution, C. Review and Critique; 3) Manuscript: A. Writing of the first draft, B. Review and Critique.

A.B.: $1 \mathrm{~A}, 1 \mathrm{~B}, 1 \mathrm{C}, 2 \mathrm{~A}, 2 \mathrm{~B}, 2 \mathrm{C}, 3 \mathrm{~A}, 3 \mathrm{~B}$; A.S.: 1A, 1B, 1C, 2A, 2B, 2C, 3A, 3B; A.P.: 1A, 2A, 2C, 3A, 3B; C.P.-L.: 1A, 1B, 1C, 2A, 2B, 2C, 3A, 3B; M.C.-M.: 1A, 2A, 2B, 2C, 3B; J.M.M.: 1B, 1C, 3B; S.A.: 1C; A.R.-M.: 2A, 2B, 2C; B.M.: 1C; P.Q.: 1C; A.C.: 1B, 1C, 3B; R.A.: 1B, 1C, 3B; P.B.: 2C, 3B; T.J.C. 1B, 1C, 3B; H.L.: 1B, 1C, 3B; L.Q.: 1B, 1C, 3B; D.S.: 1B, 1C, 3B; G.O.: 1B, 1C, 3B; J.R.: 1B, 1C.; D.R.-M.: 1C, 3B; J.C.: 1B, 1C, 3B

\section{DISCLOSURES}

Funding Sources and Conflict of Interest: This work has been performed in the framework of the FP7 project REMPARK ICT-287677, which was funded by the European Community. There are no potential conflicts of interest to report.

Financial Disclosures for the previous 12 months: No financial relationships are relevant to this manuscript.

\section{ACKNOWLEDGMENTS}

The authors would like to acknowledge all patients who participated in the pilots for their excellent and generous collaboration. We would like to acknowledge the contributions of colleagues from REMPARK Consortium.

ETHICAL COMPLIANCE STATEMENT: We confirm that we have read the Journal's position on issues involved in ethical publication and affirm that this work is consistent with those guidelines. 


\section{REFERENCES:}

1. Jankovic J. Parkinson's disease: clinical features and diagnosis. J Neurol Neurosurg Psychiatry. 2008 Apr 1;79(4):368-76.

2. Chaudhuri KR, Schapira AH. Non-motor symptoms of Parkinson's disease: dopaminergic pathophysiology and treatment. Lancet Neurol. 2009 May ;8(5):464-74.

3. Reimer J, Grabowski M, Lindvall O, Hagell P. Use and interpretation of on/off diaries in Parkinson's disease. J Neurol Neurosurg Psychiatry. 2004 Mar;75(3):396-400.

4. Goetz CG, Leurgans S, Hinson VK, Blasucci LM, Zimmerman J, Fan W, et al. Evaluating Parkinson's disease patients at home: utility of self-videotaping for objective motor, dyskinesia, and ON-OFF assessments. Mov Disord. 2008 Jul 30;23(10):1479-82.

5. Godinho C, Domingos J, Cunha G, Santos AT, Fernandes RM, Abreu D, et al. A systematic review of the characteristics and validity of monitoring technologies to assess Parkinson's disease. J Neuroeng Rehabil. 2016 Dec 12;13(1):24.

6. Sánchez-Ferro Á, Elshehabi M, Godinho C, Salkovic D, Hobert MA, Domingos J, et al. New methods for the assessment of Parkinson's disease (2005 to 2015): A systematic review. Mov Disord. 2016 Sep;31(9):1283-92.

7. Espay AJ, Bonato P, Nahab FB, Maetzler W, Dean JM, Klucken J, et al. Technology in Parkinson's disease: Challenges and opportunities. Mov Disord. 2016 Sep;31(9):127282.

8. Kubota KJ, Chen JA, Little MA. Machine learning for large-scale wearable sensor data in Parkinson's disease: Concepts, promises, pitfalls, and futures. Mov Disord [Internet]. 2016 Sep;31(9):1314-26.

9. Hoff JI, van der Meer V, van Hilten JJ. Accuracy of objective ambulatory accelerometry in detecting motor complications in patients with Parkinson disease. Clin Neuropharmacol. 2004;27(2):53-7.

10. Das S, Trutoiu L, Murai A, Alcindor D, Oh M, De la Torre F, et al. Quantitative measurement of motor symptoms in Parkinson's disease: a study with full-body motion capture data. Conf Proc . Annu Int Conf IEEE Eng Med Biol Soc IEEE Eng Med Biol Soc Annu Conf. 2011 Aug;2011:6789-92.

11. Fisher JM, Hammerla NY, Ploetz T, Andras P, Rochester L, Walker RW. Unsupervised home monitoring of Parkinson's disease motor symptoms using body-worn accelerometers. Parkinsonism Relat Disord. 2016 Dec;33:44-50.

12. Mera TO, Filipkowski DE, Riley DE, Whitney CM, Walter BL, Gunzler SA, et al. Quantitative analysis of gait and balance response to deep brain stimulation in Parkinson's disease. Gait Posture. 2013 May;38(1):109-14.

13. Cancela J, Pastorino M, Arredondo MT, Nikita KS, Villagra F, Pastor MA. Feasibility study of a wearable system based on a wireless body area network for gait assessment in Parkinson's disease patients. Sensors (Basel). 2014 Mar;14(3):4618-33.

14. Cancela J, Pastorino M, Tzallas AT, Tsipouras MG, Rigas G, Arredondo MT, et al. Wearability assessment of a wearable system for Parkinson's disease remote monitoring based on a body area network of sensors. Sensors (Basel) [Internet]. 2014 Sep 16 [cited 2017 Feb 21];14(9):17235-55.

15. Tzallas A, Tsipouras M, Rigas G, Tsalikakis D, Karvounis E, Chondrogiorgi M, et al. 
PERFORM: A System for Monitoring, Assessment and Management of Patients with Parkinson's Disease. Sensors [Internet]. 2014 Nov;14(11):21329-57.

16. Horne MK, McGregor S, Bergquist F. An Objective Fluctuation Score for Parkinson's Disease. Toft M, editor. PLoS One. 2015 Apr 30;10(4):e0124522.

17. Keijsers NLW, Horstink MWIM, Gielen SCAM. Ambulatory motor assessment in Parkinson's disease. Mov Disord. 2006 Jan;21(1):34-44.

18. Hughes AJ, Daniel SE, Kilford L, Lees AJ. Accuracy of clinical diagnosis of idiopathic Parkinson's disease: a clinico-pathological study of 100 cases. J Neurol Neurosurg Psychiatry. 1992 Mar;55(3):181-4.

19. American Psychiatric Association., American Psychiatric Association. Task Force on DSM-IV. Diagnostic and statistical manual of mental disorders : DSM-IV-TR. American Psychiatric Association; 2000. 943 p.

20. Fahn S, Marsden CD, Calne DB, Goldstein M, eds. Recent Developments in Parkinson's Disease, Vol 2. Florham Park, NJ. Macmillan Health Care Information 1987, 153-163, 293-304.

21. Hoehn MM, Yahr MD. Parkinsonism: onset, progression, and mortality. 1967. Neurology [Internet]. 1998 Feb;50(2):318 and 16 pages following.

22. Folstein MF, Folstein SE, McHugh PR. "Mini-mental state". A practical method for grading the cognitive state of patients for the clinician. J Psychiatr Res. 1975 Nov;12(3):189-98.

23. Bangor A, Kortum $P$, Miller J. Determining what individual SUS scores mean: adding an adjective rating scale. J Usability Stud. 2009;4(3):114-23.

24. Demers L, Weiss-Lambrou R, Ska B. The Quebec User Evaluation of Satisfaction with Assistive Technology (QUEST 2.0): An overview and recent progress. Technol Disabil. 2002;14(3):101-5.

25. Rodríguez-Martín D, Pérez-López C, Samà A, Cabestany J, Català A. A Wearable Inertial Measurement Unit for Long-Term Monitoring in the Dependency Care Area. Sensors. 2013 Oct 18 [cited 2017 Apr 4];13(10):14079-104.

26. Rodríguez-Molinero A, Samà A, Pérez-Martínez DA, Pérez López C, Romagosa J, Bayés À, et al. Validation of a portable device for mapping motor and gait disturbances in Parkinson's disease. JMIR mHealth uHealth. 2015 Feb;3(1):e9.

27. Pérez-López C, Samà A, Rodríguez-Martín D, Moreno-Aróstegui JM, Cabestany J, Bayes $A$, et al. Dopaminergic-induced dyskinesia assessment based on a single belt-worn accelerometer. Artif Intell Med. 2016 Feb;67:47-56.

28. Papapetropoulos SS. Patient Diaries As a Clinical Endpoint in Parkinson's Disease Clinical Trials. CNS Neurosci Ther. 2012 May;18(5):380-7.

29. Fisher JM, Hammerla NY, Rochester L, Andras P, Walker RW. Body-Worn Sensors in Parkinson's Disease: Evaluating Their Acceptability to Patients. Telemed J E Health [Internet]. 2016 Jan;22(1):63-9.

30. van Uem JMT, Maier KS, Hucker S, Scheck O, Hobert MA, Santos AT, et al. Twelve-week sensor assessment in Parkinson's disease: Impact on quality of life. Mov Disord. 2016 Sep;31(9):1337-8. 
